\title{
Prácticas de gestión de directivos universitarios: una mirada desde los principios del paradigma emergente y un camino de oportunidades por explorar*
} Management Practices of University Officers: A Look from the Principles of the Emerging Paradigm and a Path of Opportunities to be Explored

Luz Mery Chaverra Rodríguez

Universidad Católica de Oriente, Colombia

lchaverra@uco.edu.co

Elvia Patricia Arango Zuleta

Universidad Católica de Oriente, Colombia earango@uco.edu.co

Faber Andrés Alzate Ortiz

Corporación Universitaria Minuto de Dios, Bello, Colombia

faber.alzate@uniminuto.edu

\section{RESUMEN}

La mirada a la calidad universitaria muestra la necesidad de promover al interior de sus instituciones de educación superior (IES) procesos de gestión académica y administrativa más eficaces, eficientes, dinámicos, recursivos, autoorganizados y sistémicos para enfrentar las diversas situaciones que se presentan en colectivos e instituciones en las que la incertidumbre, la dispersión y el cambio son atributos emergentes en la cotidianidad. Se socializan reflexiones acerca del abordaje teórico de la investigación que le da origen, cque buscó reconocer los principios del paradigma emergente de la complejidad en las prácticas de gestión de los actuales directivos docentes universitarios de las IES. Metodológicamente, se hizo un diseño cualitativo por estar orientado a una investigación de carácter inductivo, para alcanzar comprensiones situadas y originales de la realidad de los actores implicados y de sus escenarios laborales a partir de una perspectiva holística y flexible. Se identificaron rasgos de gestión basados en los principios: autoorganización, incertidumbre, recursividad y autonomía-dependencia, y se logró inferir que parte del quehacer académico y administrativo de las IES supera la visión de una gestión clásica basada exclusivamente en la prescripción, la linealidad, el orden y la certidumbre.

PALABRAS CLAVE: directivos universitarios, paradigma emergente, prácticas de gestión universitaria, calidad educativa

\section{ABSTRACT}

The focus on university quality highlights the need to promote academic and administrative management processes within their institutions, which prove to be more effective, efficient, dynamic, recursive, self-organized and systemic in order to face the chaos, uncertainty, dispersion and change, as emergent attributes in everyday life in collectivities and institutions. In this sense, the present article socializes theoretical results of the research whose main objective was to recognize the principles of the emerging paradigm in the management practices of the current university teaching managers. Methodologically, it is a qualitative design since it was oriented to an inductive research, aiming to achieve situated and original understandings about the reality of the actors involved and their work scenarios from a holistic and flexible perspective. In this order of ideas, there were management traits identified based on four principles, namely: Self-organization, Uncertainty, Recursiveness, and Autonomy-dependency, which made possible to infer that a large part of the academic and administrative work of higher education institutions exceeds the vision of a classical management based solely on prescription, linearity, order, and certainty.

KEY WORDS: university managers, emergent paradigm, university management practices, educational quality

* Este artículo deriva de la investigación: "Los principios del paradigma emergente en las prácticas de gestión de los actuales directivos docentes universitarios: Caso Universidad Católica de Oriente y Corporación Universitaria Minuto de Dios, Uniminuto, seccional Antioquia-Chocó”. 


\section{INTRODUCCIÓN}

Hoy las instituciones de educación superior en Colombia viven momentos que las obligan a repensarse permanentemente y, con ello, a resignificar sus modos particulares de ser y actuar, teniendo en cuenta las complejidades que existen para sobrevivir en un país que, producto de sus diferentes crisis internas, poco invierte en la investigación, la innovación y la tecnología, y donde educarse profesionalmente termina siendo un privilegio de unos cuantos por lo difícil que resulta acceder a ello, dadas las actuales apuestas locales y globales por la calidad educativa que exigen a las instituciones superar sus modelos clásicos de gestión académica y administrativa, y alcanzar indicadores de inversión que, en muchos de los casos, pueden estar por encima de las capacidades financieras y logísticas disponibles.

Lo anterior puede que, desde la estrategia, lo financiero y logístico pueda resolverse en mayor o menor medida. Sin embargo, lo que en el fondo se requiere es que las prácticas humanas de las personas que constituyen la vida universitaria, es decir, la universidad en sí misma, puedan estar orientadas a consolidar un proyecto educativo que deje atrás los presupuestos clásicamente lineales y dogmáticos, en los que el centro de la obra no es la persona y su ecosistema, sino las certificaciones, los registros, el mobiliario o las cifras. Por lo tanto, un quehacer universitario centrado en las capacidades y el contexto, sin duda, sugiere desarrollar estilos de gestión orientados a principios como la autoorganización, la recursividad, la autonomíadependencia y la incertidumbre, que con seguridad nos proponen otros modos de vivir y construir el proyecto educativo universitario. Entre las razones para afirmar lo anterior se encentran:

- Las dinámicas humanas, sociales y de estructura de las instituciones y organizaciones están marcadas por las cualidades de quienes las conforman, más que por los designios o la asignación de funciones, cargos y responsabilidades. En ese sentido, no es proporcional el vínculo entre capacidades y rol o cargo; más bien, las capacidades conjugan experiencia, habilidades desarrolladas, oportunidades educativas y fortalecimiento de atributos en el medio donde se hallan las personas. 
- El despliegue de las capacidades de las personas está en concordancia con las interacciones cotidianas en los lugares donde se desenvuelven. Así que, de manera individual y colectiva, se está conectado con otros y con los objetos circundantes, de modo que las condiciones particulares de vida mantienen reciprocidad con la vida de la organización.

- Las particularidades de cada persona pueden ser valoradas de distinta manera por quienes conforman los colectivos de trabajo. Así, las singularidades que llevan a tomar distancia de quienes son vistos fuera del estándar por presentar ritmos distintos, han de ser analizadas y convertidas en oportunidad para flexibilizar procedimientos que ofrezcan a todos oportunidades de participar, aportar y enriquecerse.

- El cambio es una constante en la vida individual, colectiva y de la sociedad en general, por eso es importante, al interior de las instituciones, definir principios orientadores de actuación y, al mismo tiempo, estar dispuestos a afrontar el devenir, no para abandonar las metas, sino para reformularlas y recrearlas.

El problema que convocó la propuesta de investigación tuvo su origen en la pregunta ¿Cuáles son los principios del paradigma emergente implícitos en las prácticas de gestión de los actuales directivos docentes universitarios? Asimismo, su relación con la calidad educativa y los procesos de mejoramiento como elementos centrales en el contexto de la educación superior colombiana, situación que revela razones suficientes para caminar académicamente en la consolidación de sistemas de gestión colaborativa. La conjugación de antecedentes, de fundamentos teóricos y análisis de los autores, fueron insumo para este artículo de reflexión, cuyo objetivo principal es explorar en las prácticas de gestión de los directivos universitarios rasgos que den cuenta de algunos principios del paradigma emergente.

Explorar esas prácticas puede traer beneficios como los siguientes: a) documentar las experiencias de directivos y ponerlas en comunicación, como una reafirmación del rasgo vinculante en los procesos, aunque no siempre se compartan los escenarios donde se actúa; b) aportar a las transformaciones educativas y sociales, como señalan Morales, Sanabria y Caballero (2014, p. 190), "el cono- 
cimiento, como parte de la competitividad de las regiones, se ha convertido en un recurso esencial que se debe adquirir, formar y recrear a partir de las interacciones de los integrantes de la sociedad"; c) identificar rutas o maneras de gestionar colaborativamente, con el empoderamiento de cada participante, sin asfixiar los procesos ni subyugar la creatividad, y d) establecer nexos con instituciones del sistema escolar que propugnan por búsquedas similares, lo que al mismo tiempo constituye una alternativa para intercambiar conocimientos y potenciar otros aprendizajes.

En este sentido, los directivos universitarios pueden fundamentar sus prácticas con relación a las interacciones propiciadas y vividas, a los ambientes diseñados u optimizados y al establecimiento de relaciones que afectan el desarrollo, el aprendizaje, los desempeños y logros dentro y fuera de la organización, al igual que de su radio de acción. Al mismo tiempo, se favorece el trabajo interdisciplinar que implica reconocer y aceptar la diversidad. Interrelacionar las maneras de gestionar tiene, entre los presupuestos principales, trabar lazos de la formación disciplinar y de experiencias diferentes, desde las que se logran perspectivas distintas en la lectura de situaciones de las que se es arte y parte, y esto es común en ámbitos administrativos, académicos, pedagógicos y de proyección, puesto que parte de reconocer a las otras personas con sus potencialidades, aportes y capacidades. Así sucede siempre que a cada persona se le vea, entienda y asuma como alguien diverso, desde su propia perspectiva y su historia de vida.

Esta investigación es un llamado a superar distractores como el mejor perfil o el desempeño exclusivo, no por negar las capacidades y las diferencias personales, sino porque en una afinidad de perfiles y una rotación de roles, las funciones pueden ser aprehendidas con sus fundamentos y procedimientos; más aún, se presentan sinergias en las que tanto el rol como la función tienden a desaparecer desde las definiciones que siempre se han asignado, dado el carácter colaborativo en el ejercicio de lo asignado.

En suma, puesto que las maneras de hacer gestión atienden a la cultura, los periodos históricos y los contextos, es posible realizar el tipo de gestión que las personas e instituciones se propongan, preferiblemente, en sintonía con su razón de ser. Para dar cuenta de lo anterior, el artículo está estructurado en las siguientes partes: 
antecedentes; fundamentos teóricos de la investigación; método y resultados; hallazgos, y otras reflexiones. Se espera que este tema propicie nuevas reflexiones y alcances en quienes asumen roles de directivos docentes en las comunidades de aprendizaje y los lectores en general.

\section{ANTECEDENTES}

En la última década del siglo anterior y la primera del actual se han acentuado las tensiones que afronta la educación superior en América Latina. De ello hacen parte las economías nacionales, frente a los tratados internacionales, la pertinencia de programas y propuestas para la educación y el desarrollo de personas vs los indicadores y las evidencias para regular, sostener y controlar la permanencia dentro de un sistema educativo que, se espera, esté articulado con los sistemas de producción. No son tensiones dicótomas, ni terminadas o terminables; más bien se trata de cambios y ajustes que exigen re-estructurar la institución ante los desafíos emergentes en la economía, el conocimiento contemporáneo, la democratización, la sociedad global y la búsqueda de equilibrio democrático (Tünnermann, 2006). En esa dirección, para Díaz (2013), el reacomodo de los sistemas educativos latinoamericanos es un tema de calidad, sustentado en el cambio social e inspirado en la globalización.

De acuerdo con Acosta (1998), en las dos últimas décadas del siglo XX, las políticas de educación superior en América Latina han pasado del desarrollismo a la modernización, lo cual implica ajustes y cambios estructurales en la economía, los procesos de democratización y el reequilibrio democrático; de aquí surgen tensiones y conflictos de distinta índole, expresados en perfiles diversos, cambiantes y complejos de las personas y de las instituciones. Los desafíos y las tensiones emergentes fuerzan, en las instituciones de educación superior (IES), una agenda de adaptación paulatina en cuanto a: una nueva institucionalidad de sus establecimientos y sistemas; la gestión de compromisos, recursos y responsabilidades en la construcción de un liderazgo institucional; políticas de integración regional para fortalecer capacidades científicas y tecnológicas en la región a partir de la movilidad de estudiantes, profesores e investigadores. 
Para el mismo Acosta (1998), las instituciones están inmersas en contextos multifacéticos, constituidos por sistemas simbólicos, reglas, normativas y constructos cognitivos. En Colombia estos cambios se aprecian desde la Constitución de 1991 y finales de 1993, con un nuevo ordenamiento jurídico de la educación superior, lo cual implica flexibilidad y eficiencia en la gestión, la distribución y el manejo de los recursos, para dejar el control patrimonial y la gestión jerárquica-burocrática de los recursos. Según el autor, en los últimos años las IES han impreso otro perfil en sus estructuras y su poder bajo principios como eficiencia institucional y responsabilidad pública, lo que redunda en una nueva gobernabilidad institucional.

En los cambios de la época confluyen prácticas y búsquedas de propuestas a las situaciones institucionales y de las instituciones en general; en éstas, según Boisier (2001), desde el contexto chileno, se buscan opciones para responder a la creciente globalización, la cual incorpora reglas de juego expresadas en negociaciones, prohibiciones, acuerdos, excepciones y dinámicas que complejizan el lenguaje por medio de un léxico que requiere ser decodificado para su comprensión, hasta imponerse como un metalenguaje socializador, así como sucede con el inglés. Según él, emerge un paradigma que no es moda ni cambio en el estilo de administración; retoma de las $\mathrm{Na}$ ciones Unidas para el Desarrollo la afirmación respecto al agobio en las personas por una pérdida de bienestar, seguridad, felicidad, entre otras, e invita a avivar el sueño por una sociedad más igualitaria que fortalezca lo común, mientras se integra mejor en su propia diversidad cotidiana con un rostro más humano.

Para Boisier (2001), el conocimiento es un imperativo en cualquier persona, un eje transversal en los cambios científicos de las organizaciones y de las empresas en cuanto a las maneras de pensar el desarrollo local; coincide con Helmsing (2001) en la supremacía del aprendizaje colectivo regional entendido como

la emergencia de un conocimiento básico común y de procedimientos a lo ancho de un conjunto de firmas geográficamente próximas, lo que facilita la cooperación y la solución de problemas comunes ... el aprendizaje colectivo ... otras formas de asociatividad requieren de una fuerte dosis de capital social, en los términos 
en que ahora se entiende este concepto, vale decir, redes de cooperación permanentes, basadas en la confianza interpersonal, capaces de operar en contextos de reciprocidad difusa, más allá de relaciones familiares o amicales y orientadas a la consecución de fines legítimos (Boisier, 2001, p. 4).

Según el mismo Boisier, coexisten un conocimiento estructural y un conocimiento funcional, el primero, centrado en un saber para comprender que un territorio organizado

(llámese región, departamento, provincia, localidad, o como se desee) es una estructura de naturaleza sistémica, abierta y compleja. En la gran mayoría de los casos la región o cualquier otro segmento territorial usado para fines analíticos o políticos, es tratada mediante un enfoque que privilegia decididamente el contenedor sobre el contenido. El conocimiento funcional alude a "un cuerpo cognitivo capaz de develar la forma como el sistema anterior se articula con su entorno y cómo modela sus propios procesos de cambio, para estos efectos, el proceso de crecimiento económico y el proceso de desarrollo en el territorio" (Boisier, 2001, p. 12).

La organización del conocimiento y su funcionalidad es considerada desde Tapiero-Vásquez y García-Quiroga (2008) un paradigma sistémico procedente de la Teoría de los Sistemas Sociales (TSS) planteada por Niklas Luhmann (1998), quien aporta a una visión más interconectada y coherente en educación, más en los actuales tiempos de globalizaciones y educaciones marcados por la descentralización educativa, cuyas administraciones en Latinoamérica tienen énfasis neoliberal, expresado por medio de las contrarreformas y de la conservación del planeta a través de la alfabetización ecológica. Estos autores destacan la autorreferencialidad basada en las relaciones consigo mismo y el reconocimiento de diferencias en el entorno como clave en la configuración del actual sistema social, lo cual lleva a superar los sistemas clausurados. La sujeción de la autorreferencialidad a la coevolución equivale a una autopoiesis, desde la que se reemplaza el interés en el número de partes y sus relaciones, por el número de diferencias operativas usables dentro del sistema; 
el diseño y el control suplidos por la sensibilidad frente al entorno, la capacidad del sistema para autoproducir e interpenetrar, según su estabilidad dinámica y la autonomía, así, en

la teoría general de los sistemas y los sistemas no existe un sentido unívoco, por no ser los sistemas una característica preexistente al hombre, sino constructos culturales que se ajustan ... La construcción de sistemas sociales es un método de la reducción de la complejidad, por mediar entre la escasa capacidad del hombre para elaborar de manera consciente sus vivencias y el mundo de extrema complejidad que vive (Tapiero y García, 2008, p. 216).

Se lee de los biólogos Humberto Maturana y Francisco Varela (citados en Razeto-Barry y Ramos-Jiliberto, 2013, p. 31) que el significado de autopoiesis se refiere a

una máquina autopoiética organizada ... como un sistema de procesos de producción de componentes concatenados de tal manera que producen componentes que: i) generan los procesos (relaciones) de producción que los producen a través de sus continuas interacciones y transformaciones, y ii) constituyen a la máquina como una unidad en el espacio físico.

Respecto a la coexistencia paradigmática, Tapiero y García (2008) afirman que Luhmann (1998) desmitificó la diversidad estructuralismo-funcionalismo, asimiló la teoría de la estructura como la teoría del lenguaje, la teoría del lenguaje como la teoría de los textos, la teoría de los textos como la teoría de la acción y por medio de éstas reconstruyó la teoría de la acción. La TSS se diferencia de la teoría de los sistemas psíquicos, al ser considerada un método para mediar entre la exagerada complejidad del mundo y la escasa capacidad humana para elaborar las vivencias.

De acuerdo con Ramírez (2012) se asiste a un impulso mundial con un alto contenido político y económico para promover el concepto de gobierno abierto, basado en apertura, transparencia, colaboración y participación, rasgos éstos de un paradigma que emerge con la pretensión de responder ante unos modelos agotados dentro de un mundo que está cambiando radical y vertiginosamente. Este 
paradigma emergente constituye una nueva institucionalidad y unas modificaciones hacia dentro y hacia fuera con proporciones que implican cambios radicales para avanzar de niveles básicos hacia niveles más complejos, "donde los ciudadanos y el gobierno co-diseñan, cocrean, co-producen, bajo una capa de nuevas prácticas inspiradas en la apertura, la transparencia, la participación y la colaboración" (Ramírez, 2012, p. 21). El autor señala en los ecosistemas abiertos una clave para los cambios gubernamentales y administrativos y plantea el gobierno abierto como la "transparencia de las acciones, la accesibilidad en los servicios públicos e información, y la capacidad de respuesta del gobierno a las nuevas ideas, demandas y necesidades" (Ramírez, 2012, p. 6); destaca la importancia concedida por la OCDE a un gobierno abierto y sostenible como una

plataforma de base para implantar sólidos marcos jurídicos, institucionales y políticos que rijan el acceso a la información, la consulta y la participación pública lo que contribuiría a mejorar el diseño y formulación de las políticas públicas, luchar contra la corrupción e incrementar la confianza de la sociedad en el sector público ... lograr una mayor implicación ciudadana en la elaboración de las políticas y la promoción del buen gobierno ... fomentar la apertura, y una buena inversión para mejorar la elaboración de las políticas públicas (Ramírez, 2012, pp. 205-206).

Navarro (1997) reflexiona el paso de un paradigma de gerencia a un paradigma de gerencia pública y a otro de gerencia social, con la pretensión de promover un trabajo gerencial lo más efectivo posible, según el entorno y las nuevas realidades organizacionales. Para él, el "nuevo paradigma de emergencia privilegia el empowerment (facultar a los recursos inteligentes), el liderazgo, el trabajo en equipo y la participación de todos los miembros de la organización en la solución de los problemas" (Navarro, 1997, p. 3). La gerencia pública busca incorporar un espíritu empresarial e implica una ruptura epistemológica con la cultura del memo, yo ordeno, yo cambio, yo mando, mientras la gerencia social busca responder a las deficiencias sociales, estructurales, institucionales e históricas; sin embargo, "el crecimiento económico, por sí solo, no asegura el desarrollo social y por lo tanto el crecimiento económico que no tenga como norte 
el desarrollo de ser humano, no sólo es antiético, sino que carece de sentido" (Navarro, 1997, p. 9). El autor destaca la importancia del cliente a quien se diseñan y dirigen los servicios, sin que esto sea sinónimo de privatización.

Para Domínguez (2001), las particularidades de la época actual son un continuum en la generación de conocimiento por parte de las organizaciones educativas, lo cual trae afectaciones culturales y repercute en las dimensiones de la organización. Su planteamiento es un llamado para que las organizaciones sean inteligentes y aprendan, lo que demanda acciones que tienden a volverse corrientes de índole colaborativa, participativa y democrática, lo cual exige considerar la organización como campo científico, que supera el carácter eficientista, en privilegio de posturas interpretativas simbólicas y culturales. Lo anterior, según Bolívar (2010), sugiere repensar las formas de gestionar las organizaciones educativas, en el sentido de generar en estas condiciones que promuevan el desarrollo de las posibilidades y las motivaciones de las personas que la conforman, así como el fomento del trabajo en equipo y la cultura colaborativa.

Los cambios afrontados dan cabida a la lectura de Casassus (2008) quien, al reconocer las raíces etimológicas del término gestión, establece diferencias con lo que se ha generalizado como administración, y asocia las prácticas administrativas verticales con las herencias de Platón, dada la importancia atribuida al establecimiento claro y prescriptivo de procedimientos y acciones a seguir, estilo este manifiesto, por ejemplo, en los controles administrativos, la consecución de objetivos, el alcance de resultados y la búsqueda de eficiencia. Reconoce en Aristóteles la aceptación de movimientos, dinámicas y cambios circunstanciales que no permiten preestablecer y prescribir lo que no se sabe cómo sucederá. Según su planteamiento, en el estilo de Platón pueden situarse prácticas y concepciones administrativas como las de Taylor (1911), quien consideró la doble mirada consistente en la racionalización operativa por parte de los administradores y el interés económico por parte de los trabajadores, y Fayol (1931) quien imprime el carácter racional a la función del administrador; mientras en la propuesta de Aristóteles encaja Mayo (1977), quien imprime a la administración el sello de las relaciones humanas, privilegiando las motivaciones de los trabajadores. 
Esas herencias se actualizan en dinámicas que llevan a las IES a buscar alternativas para adaptarse gradualmente a los continuos desafíos, lo que conlleva a: una nueva institucionalidad de los establecimientos de educación superior; repensarse ante la sociedad actual; integrarse plenamente con la sociedad; promover el diálogo constructivo, y hacerse responsable social: "Al mismo tiempo implica asumir un concepto de pertinencia social integral; es decir, estar plenamente consciente de que la relevancia de su trabajo será evaluada en función de su compromiso social y de que éste genere beneficios concretos" (Tünnermann, 2006, p. 6). Son desafíos: la construcción de un liderazgo institucional; la gestión de compromisos y recursos para el desarrollo de la misión; el impulso y la concreción de políticas de integración regional; la creación de redes de conocimiento dinamizadas por la apertura a distintas opciones; la movilidad de estudiantes, investigadores y docentes; la innovación, el desarrollo de capacidades y el posicionamiento por la generación de conocimiento, productos científicos y tecnológicos al servicio de la comunidad del radio de acción y de la sociedad en general. Este mundo de constante cambio da lugar a la educación permanente como respuesta pedagógica y estratégica, que permita aprender a aprender a lo largo de la vida.

Chaverra y Alzate (2014, p. 2) hacen ver la importancia de gestionar el conocimiento con un valor duradero, pertinente y calificado en todos los niveles. Para lograrlo, se precisa de los "procesos de resignificación y mejoramiento institucional, desde la perspectiva del desempeńo ... anclado en la promoción e inclusión de aspectos como el respeto por la diferencia, el trabajo en equipo, la ética profesional, el empoderamiento y la confianza recíproca en la cultura/clima organizacional". Los mismos autores estiman posible "concebir las instituciones de educación superior como organizaciones vivas, autorreguladas, con capacidad para autogestionarse y entrar en contacto vital con el entorno que las rodea, creando tramas/urdimbres bioaprendientes, en función de las dimensiones epistemológicas y ontológicas del conocimiento que son producto emergente de la investigación, la docencia y la extensión que, social y científicamente, las caracteriza" (Chaverra y Alzate, 2014, p. 4).

La somera pesquisa deja ver cómo el tema de la gestión en las IES continúa ante las tensiones de ajustarse a estándares y buscar calidad, 
proclamar autonomía y enfatizar heteronomía, abogar por la formación y subrayar la instrucción. Las pautas para proceder y las medidas centradas en indicadores asfixian la riqueza de las prácticas en las distintas áreas de gestión, al parametrizar las funciones a tal grado, que la iniciativa, la participación voluntaria y la creatividad se cohíben ante experiencias de crecimiento, contribución y empoderamiento, y cuanto surge en el día a día queda sin registro sistemático.

El breve rastreo confirma la novedad del tema de la investigación que da origen a este trabajo, cuyo objetivo fue reconocer los principios del paradigma emergente en las prácticas de gestión de los actuales directivos docentes universitarios. Se tomaron como caso de estudio la Universidad Católica de Oriente y la Corporación Universitaria Minuto de Dios, ambas de carácter privado confesional, situadas en el Departamento de Antioquia, Colombia (del proyecto de investigación original). En estas instituciones son claras las tensiones por ajustarse a estándares y buscar calidad, proclamar autonomía en un mundo heterónomo, considerar competencias instruccionales. De estas IES puede generalizarse, para Colombia, que la educación superior afronta la encrucijada de autorregulación, mezclada en procesos de control rígidos y estandarizados, en las apuestas por un carácter diferencial y por una diversidad humana tendiente a estar homogenizada en los currículos, así como en los demás procesos y las áreas de gestión.

Lo expuesto lleva a considerar cómo las IES en Colombia, abocadas por las dinámicas del contexto actual en la búsqueda desaforada de indicadores y evidencias para ser valoradas y consideradas de calidad en sus ofertas, pueden incurrir en la invisibilidad de capacidades y talentos diversos de personas que no sólo aportan a esos intereses, sino que enriquecen los procesos institucionales.

Ese panorama es común en distintas gestiones, pero el centro de este proyecto es la gestión en directivos universitarios, quienes con frecuencia afrontan los roles y las responsabilidades propias de un cargo que deja poco tiempo para compartir en comunidad las experiencias, puesto que el afán de rendir cuentas está sujeto a la relación de productos preestablecidos o determinados; igualmente, se someten a responder por las dinámicas de quienes están bajo su gestión, ajenos a los testimonios de vivencias para su consecución, extraños incluso a sus propias lógicas y dinámicas. Desde otra perspectiva, 
tienen capacidad de decisión y de actuación con una autonomía y una independencia finalmente dependientes de las decisiones en instancias superiores.

Con el proyecto de investigación, Los Principios del Paradigma Emergente en las Prácticas de Gestión de los actuales Directivos Docentes Universitarios, se buscó responder a las dinámicas y los propósitos de dos instituciones investigadoras que abrigan el deseo de "aprender a ser, a conocer y a desear seguir aprendiendo; el aprender a hacer y a convivir en el respeto y en el amor a la diferencia, la solidaridad y la democracia” (Plan de Desarrollo Institucional-UCO 2016-2025, s/p); también el deseo de "Fortalecer la investigación orientada al Desarrollo Humano y el Desarrollo Social integral y Sostenible y la Innovación Social, generando soluciones innovadoras a problemáticas sociales concretas" (UNIMINUTO, Compendio de Investigaciones, 2015, p.13).

\section{FUNDAMENTOS TEÓRICOS DE LA INVESTIGACIÓN}

La pesquisa de experiencias relacionadas con el tema de investigación, al igual que la fundamentación teórica, llevaron a las nociones que se presentan a continuación, combinadas con reflexiones procedentes de vicisitudes en la cotidianidad de los directivos universitarios, a quienes además, se hace referencia como directivos docentes, atendiendo a que son, en su mayoría, docentes con encargo o comisión administrativa interna, lo que les lleva a compaginar la docencia y la vida académica en general con los procesos de administración.

Para referirse a las prácticas de gestión se parte de señalar que a la práctica se atribuyen distintos usos y definiciones; por ejemplo, comúnmente es considerada aplicación de teorías o conocimientos; también la experiencia acumulada en un campo de saber doxa o científico. Desde el paradigma emergente la práctica de los directivos se abre a los acontecimientos porque nada es inalterable; la mecanización de procedimientos cede ante situaciones que no siempre pueden resolverse de la misma manera ni en el mismo tiempo (Jara, 2004). Puesto que se trata de prácticas de gestión, según Cabanelas (1993), el término es referido al acto de desempeñar una función administrativa de negocios ajenos. Dicha definición retoma el concepto romano negotiorum o persona que se dedica, por su cuenta y sin encargo, a administrar asuntos de un ausente. 
A partir de la definición anterior se puede apreciar: a) un conjunto de acciones realizadas por alguien para diligenciar determinada misión hacia un fin, como es el caso de los directivos docentes; b) la recursividad asociada a una expresión de creatividad en perspectiva cultural, cualidad aquella que agrega valor a la actividad (Verlee, 2008); c) la negociación como clave en un proceso de diálogo; d) la gestión como un proceso vinculado a estructuras de la sociedad reflejadas en la institución y la respectiva autonomía otorgada por ley, y e) la actuación a nombre de alguien menor, afirmación que lleva a preguntarse, ¿en qué está representada la minoría? ¿De qué gestión se habla cuando unas personas deciden por otras o cuando, teniendo condiciones para gestionar, se opta por ajustarse a las decisiones de otros?

Es común escuchar, cuando se habla de directivo, que se alude a quien es responsable por un establecimiento o que, entre sus actividades, dirige, ordena, lidera, encabeza o administra, coordina, controla y maneja un colectivo, unos procesos o unas cosas. De ahí, dos maneras usuales de nombrar: 1) de director, que respondía por un centro educativo donde se atendía la educación de los grados primero a quinto de ciclo, se pasó a rector, quien es el responsable del direccionamiento, la marcha y los resultados de la educación básica ofertada en los grados de preescolar a noveno, en muchos casos en educación media, ${ }^{1}$ y del establecimiento en general. El número de estudiantes en una institución de educación básica dependiente directamente del Estado es un criterio que usa el Ministerio de Educación Nacional para determinar por medio de las Secretarías de Educación departamentales y locales el número de coordinadores que dan direccionamiento académico y en convivencia en el respectivo establecimiento, en apoyo a las funciones del rector. 2) Con el término directivo se nombra, en las instancias administrativas del sistema de educación nacional, a quienes son docentes y, por necesidades locales -distancia del casco urbano, condiciones geopolíticas, dispersión y número de estudiantes, etc.-, además, direccionan las áreas y los procesos del establecimiento educativo.

\footnotetext{
${ }^{1}$ En Colombia, el sistema de educación formal concibe preescolar como el primer grado y desde primero hasta quinto como educación básica ciclo primaria y desde sexto a noveno es educación básica ciclo secundaria, clasificando cada ciclo por niveles y grados. Con los denominados grados 10 y 11 culmina la educación de esos dos ciclos o educación básica, y durante su curso suele realizarse alguna formación vocacional, que se puede certificar como una formación técnica.
} 
El anterior significado tiene síntesis en la siguiente afirmación: "director es el regente de las instituciones de enseñanza inicial y primaria ... De esta manera, tanto en rector como en director está presente la idea de conducción, acorde con su rol de organizadores institucionales" (Castellano y Mársico, 1995, p. 11). Directivo se ha utilizado como apelativo para referir al director en educación básica, constar su importancia en la vida escolar y en la eficacia de sus procesos, al corresponderle "organizar el funcionamiento del centro escolar para lograr los objetivos institucionales, articular la organización, la planificación, la gestión de los recursos, el seguimiento de las actividades educativas, la evaluación de los aprendizajes y las relaciones con la comunidad educativa" (Aguilera, 2011, p. 11).

Las nociones planteadas generan inquietudes en relación con la organización de las universidades, las funciones asignadas según las jerarquías, el alcance del carácter institucional y la conjugación de sistemas que constituyen los enfoques desde los cuales actúan. En las IES se cuenta con las figuras de vicerrector académico y director académico, maneras éstas de nombrar con significados conceptuales distintos, pero con responsabilidades y funciones difusas en la práctica. Debido al interés de este trabajo, el énfasis se hace en la figura director o directivo.

En cuanto a director académico en educación superior, se presenta coincidencia respecto a la escasa información del significado, mientras se halla material alusivo a las funciones, responsabilidades, habilidades y competencias que debe tener. Afirma Elizondo (2011) que sólo algunas universidades superiores describen su figura sin hacer referencia a su papel. Se enfatiza en funciones como la acreditación nacional e internacional de programas y de la institución, diseño de programas académicos, promoción de valores y de ética, investigación educativa sobre las competencias de ingreso y de egreso conforme a los planes de estudio; difusión de tecnología educativa, planeación y seguimiento a objetivos estratégicos, entre otras. Las principales referencias son acerca de actividades y funciones procedentes de investigación en ese tema, sintetizables en que para el directivo universitario sea posible partir de la comprensión y aprehensión de sus roles y funciones, para "contribuir de forma más participativa, comprometida, motivada y creativa al logro de la visión de la educación superior en la actualidad, caracterizada por 
la búsqueda de la pertinencia y la calidad" (Elizondo, 2011, pp. 207-208). Aveiga y Marín (2013), a su vez, señalan que el directivo universitario es responsable de optimizar y lograr la pertinencia del proceso formativo y requiere una cultura general y específica de una profesión definida.

La importancia concedida a ese cargo y sus dimensiones amerita analizar si, en efecto, es tal la operatividad centrada en actividades, si ha girado de fondo y de forma el componente teleológico de las instituciones, si hay un mayor acoplamiento con el engranaje productivo en el país o si hay una tecnificación de la proclamada formación integral, entre otros supuestos.

Al indagar el significado del término docente se halla que es denominado, indistintamente, profesional, profesor o maestro conducente de la enseñanza y del aprendizaje en determinado nivel educativo. Docente es quien asume la tarea de educar. Es un vocablo latino que da lugar a docente y docencia, derivados del verbo doceo, que significa aceptar y "por su valor causativo, toma el sentido de hacer aceptar, de donde hacer aprender y por lo tanto enseñar. Se opone entonces a discere que, sin valor causativo, conserva el sentido de aceptar, acoger, y por lo tanto aprender" (Castellano y Mársico, 1995, p. 13).

Los directivos universitarios, por una parte, tienen el encargo de aceptar las disposiciones de las instituciones para que los equipos de colaboradores realicen lo propio de sus cargos y funciones; al mismo tiempo, cuando son docentes en comisión administrativa (por encargo y necesidad), velan por la calidad y el cumplimiento de los aspectos académicos y administrativos. En cuanto a la profesión docente, la escasa remuneración influye en su subvaloración, en contraste con las responsabilidades que asume. Priman las nociones centradas en su papel, no en quién es.

El paradigma emergente aludido en el título es el denominado por algunos autores, paradigma de la complejidad, el cual se aparta del modelo occidental "construido a partir de una comprensión del mundo lineal, producto de una matriz de pensamiento newtoniano clásico que prioriza la estabilidad, las estructuras y el cambio se entiende como un evento anómalo" (Tsoukas, mencionado en Aguerrondo, 2014, p. 564). Como una ampliación de esa postura, se toma la mirada de Ander Egg (mencionado en Chacón, 2014, p. 
158), para quien este paradigma supera el pensamiento simple, reduccionista, unidimensional, mutilador de la realidad, programador y controlador de lo que considera firme, cierto y estable, seguro, para lograr lógicas caracterizadas por "asociar lo que está desunido, concebir la multidimensionalidad de toda realidad antroposocial, va en vías de diseñar estrategias para abordar lo irreversible, lo aleatorio y lo cualitativo".

Desde el proyecto que da origen a este artículo, se comparte la complejidad como: a) un discurso de la ciencia; b) un discurso general que, en lugar de respuestas, ubica frente al desafío de entender la condición cambiante de la realidad de donde siempre emergen cosas nuevas (Morin, 1999); c) un cambio de estilo en todos los aspectos, y ésa es una meta fundamental; d) emergencia de procesos en los que convergen el azar, la indeterminación y la incertidumbre (Morin, Roger-Ciurana y Motta, 2003).

Gestionar desde los principios del paradigma emergente implica otras maneras de concebir la administración al interior de las universidades, lo que conduce a buscar siempre caminos alternativos tendientes a superar las lógicas y maneras de vivir fija y linealmente para marchar de una manera mucho más abierta, flexible, holística, participativa; es decir "una transformación fundamental de nuestros pensamientos, de nuestras percepciones y de nuestros valores" (Capra, 1998, p. 10). Gestionar, lleva a entender, con Bauman (2000, p. 28), que los humanos "rara vez están satisfechos con sus resultados y que siempre están deseosos de rectificarlos”, lo cual es entendido por Payán (2000, p. 9) como "mecanismo de autoemergencias no lineales" y por Lipton (2007, p. 23), como la importancia de estar "decididos a no dejarse vencer por el sistema que estandariza, normaliza y determina".

Los significados hasta aquí compartidos iluminaron el reconocimiento de principios del paradigma emergente en las prácticas de los directivos universitarios. Los procedimientos utilizados y los hallazgos hacen parte de otro texto. En este artículo se plasman los significados de algunos principios hallados; se trata de: la incertidumbre y la indeterminabilidad; la recursividad, y la autonomía-dependencia. 


\section{PRINCIPIO DE INCERTIDUMBRE Y DE INDETERMINABILIDAD}

En Bartra (2011), quien retoma a Heisenberg, defensor de la no predeterminación de la existencia, se puede tener un primer acercamiento al concepto de incertidumbre e indeterminabilidad, lo que significa que, ya sea por la diversidad de sujetos, circunstancias o situaciones, se pueden afectar los procesos y las metas organizacionales, ocasionando modificaciones en lo que se pensaba y se tenía previsto. Lo anterior, de acuerdo con Ball (2013), indica que, constantemente, la organización está abocada a cuestionar si lo que proyecta y realiza es suficiente, correcto y satisfactorio para retomar y reinventar cada vez la dinámica organizacional.

En el paradigma emergente de la complejidad, la incertidumbre es la certitud que ni la materia, ni la naturaleza, ni las personas, los grupos o comunidades son realidades estáticas ni lineales: "Hay que aprender a enfrentar la incertidumbre, puesto que vivimos una época cambiante donde los valores son ambivalentes" (Morin, 1999, p. 53); esto supone considerar única certeza la condición de indeterminabilidad e incertidumbre del universo, de las personas y de los grupos sociales. En la línea de la gestión educativa, supone la conciencia de que siempre se debe tener abierta la posibilidad para replantear, reformular, recrear la vida de la organización (Pigem, 1991).

Lo anterior no significa falta de planificación, ligereza en la gestión ni desesperanza o actitud pasiva, sino desapego a la rigidez y apertura a lo posible; planear para "llegar a un final pensado, imaginado y al mismo tiempo insólito, imprevisto y errante" (Morin, Roger-Ciurana y Motta, 2003, p. 13). Al decir de Calvo (2009), superar lo establecido e incursionar en lo inimaginable, lo incierto e imprevisto. Promover, en la vida organizacional, una experiencia humana, que integra el lenguaje, la cultura, la emoción de las personas; en palabras de Gutiérrez (2008, p. 173), "no se debe rutinaria y cronométricamente perseguir objetivos y metas, sino realizarse dando significación plena a cada momento presente. No se trata tanto de lograr fines, sino de crearlos”. 


\section{PRINCIPIO RECURSIVO: INCLUYE LA AUTO-PRODUCCIÓN Y LA AUTO-ORGANIZACIÓN}

Para explicar este principio resulta oportuno acudir a Mussa, Morales y Martínez (2011), para quienes, en un proceso recursivo, los productos y efectos son al mismo tiempo origen y productor de aquello que los produce; en este mismo sentido, para Guarnizo (2003), todo organismo y toda organización social es producto de las interacciones de los elementos o individuos que los conforman; por eso son sistemas y, a su vez, retroactúan, se reproducen y desarrollan en el mismo, de tal forma que cada parte se hace necesaria en los procesos de autoproducción y autoorganización del todo, lo que, en términos de Ballesteros (2011), refiriéndose a la acción social, significa que los productos en cada proceso son al mismo tiempo causa y efecto.

La organización desde este principio se dinamiza a través de la promoción e intensificación del diálogo, la recreación de relaciones, de los procesos y las estrategias para permitir a cada persona implicarse como en una danza armónica; es, para Lederman y Teresi (1996, p. 264), "un bazar de frenética actividad". Al favorecer "la expansión de la autonomía personal respecto a las instituciones" (Ugarte, 2007, p. 48), se propician formas de armonizar el colectivo, posibilitar la interacción amigable, vital, el despliegue personal y colectivo. Según Bateson (1991, p. 66): “cada vez que comenzamos insistiendo con demasiada energía en el operacionalismo, perdemos algo de la capacidad para pensar pensamientos nuevos".

Elizalde (2003, p. 38) habla de organizaciones que orientan sus procesos y dinámicas desde lógicas de conservación sin apertura al cambio; señala cómo los argumentos sobre real y realidad son usados "cada vez que intentamos forzar a otro ser humano, sin usar la fuerza, a hacer algo que queremos, y que el otro no hará espontáneamente". Desde otra perspectiva, Gutiérrez (2006, p. 13) invita a una gestión que "se vuelca a los otros y a lo otro"; en palabras de Novo (2007, p. 39), "pasar del predominio de las historias construidas por otros a valor de las historias que se construyen". Siguiendo a Assman (2002), es hacer de los colectivos nichos vitales de despliegue en los que los participantes no sean ubicados como observadores, separados de un universo que les es ajeno. Ésas son señales de una organi- 
zación, según Morin (1999, p. 37), reconocedora de "las múltiples facetas de lo humano", en las que como lo indica Maturana (1992), se logran procesos de humanización al reconocer, incluir y acoger la diferencia.

Flores (1997) señala la importancia de la comunicación, elemento en el que también enfatizan Boff y Muraro (2004, p. 203): "En las organizaciones emergentes, la palabra empeñada vale, la confianza es todo, nadie es dueño de la verdad ... la coexistencia se funda en el amor, en la proximidad, en la conversación reflexiva y en la capacidad de consensuar". Estos autores coinciden en la necesidad del diálogo, concebido por Bohm (1997) como encuentro directo y cara a cara, en el que se tejen relaciones que promueven y favorecen la participación.

Principio de la autonomía/dependencia: plantea la autonomía de los seres humanos y, a la vez, su dependencia de los otros, del medio, de los grupos a los que pertenece

Para alcanzar un nivel de comprensión de lo que significa este principio, es preciso acudir a Morin, Roger-Ciurana y Motta (2003), quienes retoman la idea de procesos auto-eco-organizacionales, a partir de los cuales no hay posibilidad de autonomía sin múltiples dependencias, lo que, desde Columbie (2012, p. 3), se entiende como "la tendencia natural de los seres humanos y de las organizaciones a las que pertenece, de autoorganizarse a partir de las relaciones intrincadas entre las partes para formar sistemas en los que se esperan procesos de adaptación como condición para tener un 'todo ordenado'", y que Tronto (mencionado en Cerri, 2015) concibe como imperativo en toda organización, por lo que se precisa atender la necesidad de ocuparse del cuidado de sí mismo y de los otros, condición práctica en las vivencias humanas.

Sobre la base de las consideraciones anteriores, se puede entender que, desde el paradigma emergente de la complejidad, autonomía y dependencia son inseparables desde el paradigma emergente, puesto que toda organización está siempre conformada por elementos o actores que dependen unos de otros. En el paradigma emergente este principio convoca a entender el sentido de las interrelaciones, conexiones e interdependencias, fundamentales a toda existencia y organización. Afirma Capra (1998, p. 41) que el mundo es "como una unidad indivisible y dinámica”, de allí su teoría acerca de la 
trama de la vida referida a las interconexiones entre todos los seres. Al respecto Morin (1999, p. 33) señala que todo desarrollo humano es resultado del "conjunto de las autonomías individuales, de las participaciones comunitarias", y Najmanovich (2008, p. 24) invita a conformar comunidades, es decir, "unidades heterogéneas"; comunidades, según Eisler (1998, p. 109), "donde verdaderamente se valore la diversidad, incluyendo las nuevas identidades de género, raza, religión o etnia”.

\section{MÉTODOS Y RESULTADOS}

Con esta investigación cualitativa e inductiva se pretendió alcanzar comprensiones situadas de la realidad de los actores implicados y de los escenarios universitarios donde se desenvuelven. Entre ambas instituciones se identificaron 26 directivos que ocupaban cargos en los cuales su gestión articulaba, paralelamente, actividades académicas y administrativas; sin embargo, los sujetos participantes fueron 20 directivos docentes con funciones administrativas, en encargo o comisión, adscritos a las IES (Corporación Universitaria Minuto de Dios, 2015, y Universidad Católica de Oriente, 2016), que cumplieron con las siguientes dos condiciones: a) tener por lo menos un año de experiencia en el cargo dentro de la institución y b) haber liderado algún proceso académico-administrativo como la adquisición o renovación de un registro calificado, la acreditación, autoevaluación, investigaciones o similares.

Con estas personas se implementaron entrevistas semiestructuradas, a las cuales se dio tratamiento con Atlas Ti. Atendiendo a la pregunta de investigación, el principal tema interrogado giró en torno a tópicos como los siguientes: atención a imprevistos humanos, académicos y administrativos; reconocimiento de capacidades y potencialidades en los colaboradores o miembros de los equipos de trabajo; potenciación de ideas que emergen de colaboradores y su materialización en proyectos colectivos pertinentes a la institución; propiciamiento de espacios que permitan superar lo laboral para compartir otros rasgos humanos; importancia de la comunicación para el entendimiento; maneras de afrontar las contradicciones y oposiciones de los colaboradores ante ciertas directrices. 
La manera de allegar los datos y transformarlos en información se caracterizó por: a) las indagaciones a los sujetos participantes que no constituyen la totalidad de la población de las universidades investigadoras $\mathrm{y}$, menos aun, el total de docentes de las universidades; sin embargo, sus apreciaciones y aportes fueron de sumo valor; b) al entrevistar a los participantes, éstos podían reconstruir episodios de sus prácticas de gestión y durante esa conversación se realizaban someros análisis relacionados con sus actitudes y comportamientos ante lo descrito; c) la información de los datos -con frecuencia, palabras clave- y apoyo en la transcripción de grabaciones, dio paso a la información, la cual se introdujo en Atlas Ti y, de acuerdo con lo arrojado por esta herramienta, se procedió a su clasificación y a la elaboración de mapas semánticos que son contenido de otro escrito. Se cotejó la información obtenida del trabajo empírico con el marco referencial y de ahí se explicitaron, como una generalización, los principios del paradigma emergente presentes en las prácticas de gestión de los directivos universitarios.

Temáticamente, en esta oportunidad se plantean algunas de las emergencias asociadas con los principios del paradigma y, debido a que es un tema poco abordado, en este estudio de caso exploratorio se aprecian distintas perspectivas, como se recuenta en el último apartado del artículo.

\section{Hallazgos}

Para llegar a los resultados de la investigación, se introdujeron en Atlas Ti textos clave de cada grupo participante y, enseguida, se efectuó un proceso por agrupación, reducción y exclusión hasta obtener redes semánticas que hacen parte de otro texto. Sin embargo, para este artículo se tomaron algunas respuestas o fragmentos de las mismas, que se constituyen en núcleo de rasgos primordiales en los principios del paradigma que emergen.

\section{Interés en las personas}

Lo que emerge y su relevancia atañe a la persona y la concepción que se tiene de la misma. Gestionar es vislumbrar caminos que pueden ser inciertos. Las cosas son hechura de las personas. El conflicto, su tramitación y alternativa de solución están sujetos al devenir, cuyo protagonista es cada persona en medio de las circunstancias que 
particularizan el contexto y su historicidad. Frente a esos rasgos, la apertura y la flexibilidad son cualidades que no riñen con los cargos directivos administrativos, pero no están explicitadas dentro de los parámetros institucionales para efectuar esas prácticas.

Muchas veces las reuniones se abren y terminan hablando del sentir: cómo se sintieron, cómo se sienten. Si a mí me vienen con un problema yo me apropio del problema ... trato de leer la angustia de esa persona y no me quedo tranquilo hasta que esto es solucionado (Informante 3).

En el día a día de un Decano hay situaciones previsibles e imprevisibles y muchas casuísticas; situaciones personales, a veces, dramáticas, etc. ... Otro elemento es el elemento humano (Informante 6). Siempre el paciente, el estudiante requiere atención en primer lugar (Informante 15).

El interés en las personas alcanza la inquietud por sus cuestionamientos, apuestas, pensamientos y emociones dentro del trabajo y fuera del mismo; de ese modo, son identificados estilos particulares en las prácticas de los colaboradores al interior de los equipos de trabajo y se perfilan los cargos según las capacidades. Son expresiones de interés de los directivos docentes: saludar a los colaboradores y coequiperos para hacerse una idea del panorama de trabajo, propiciar espacios distintos a los académicos para interrelacionarse e interactuar; enterarse de lo que consideran básico en las dinámicas sociofamiliares de los colaboradores, sin interferir ni generar intromisión; buscar puntos de apoyo entre los integrantes de los colectivos de trabajo.

Para quienes no son coequiperos también hay atención e interés. Así se desprende de quienes priorizan a los estudiantes con sus vicisitudes por encima de los compromisos administrativos; igualmente, en quienes pretenden hacer de la academia un espacio de encuentro humano, no sólo de propiciar ambientes para la apropiación de contenidos.

Con relación a los equipos colaboradores, destaca la importancia de directivos docentes que, antes de iniciar la sesión de cada día, saludan, invitan a orar, cuentan lo que harán durante la jornada y la 
manera de hacerlo. No es interés del grupo investigador, a partir de las apreciaciones sintetizadas, ahondar en prácticas religiosas, sino en generalizar rutinas que, al responder a las expectativas de los participantes, propician atmósferas para la realización del trabajo y para el libre fluir dentro del mismo.

En el interés subyace la disposición como apertura ante los otros y ante el devenir; por cuanto al indagar estados anímicos, queda abierta la posibilidad de acercarse a situaciones e imprevistos que intervienen en el rendimiento productivo, pero también aproximarse a una condición humana para entenderla, acompañarla y cooperar con ella desde el respeto, en la búsqueda de alternativas para marchar juntos hacia los logros propuestos. Este rasgo revela una gestión en la que los planes con una ruta para conservar y tener presente la meta a alcanzar, no hace indispensable seguir un solo y mismo camino.

Empiezo a conocerlos, a identificar, a verlos en el patio ... Soy rígido y seco en la relación, pero es una relación de mucha disciplina, acogedora, de respeto, de llegar a entender qué estamos haciendo, por qué lo estamos haciendo y para qué lo estamos haciendo (Informante 8).

Percatarse de los retos implica reconocer a las personas, sus capacidades, conocimientos, potencialidades y expectativas, acción que, al superar el mecanicismo y cumplimiento de funciones, favorece el empoderamiento. Para ello es importante, en el directivo:

- La observación de las personas en distintos momentos, circunstancias, horarios y espacios; de ese modo, se hace una imagen de las personas con quienes comparte.

- La comunicación oportuna y clara en lo tocante a las intencionalidades del encuentro académico, así como en la identificación de expectativas de quienes son los participantes.

- La escucha respetuosa de quienes están involucrados en el proceso de formación.

- La autoobservación y su contraste con la observación de otros para aproximarse a lo que el directivo puede ser y reflejar ante los otros. 
Ante este testimonio, los coequiperos destacan la importancia de una visión clara y expresa en quien detenta el rol directivo; de igual manera, señalan la importancia de ser considerados en los procesos de diseño, planeación y desarrollo de las actividades de formación. Sostienen que ésa es una forma personal de seguir aprendiendo.

Programa de educación ambiental con procesos reflexivos ... para que salgan estrategias pero que sean de interés, motivantes para las personas del campo, incluso, que salgan de las mismas comunidades (Informante 13).

Este testimonio refleja la importancia de la reflexión personal en el trabajo conjunto, acción que se constituye en motor de análisis, decisiones y acciones en las que se implica el equipo de colaboradores. Se trata de un involucramiento propiciado por la actitud de quien lidera, toda vez que se dispone a escuchar iniciativas, examinar posibilidades, considerar riesgos y atreverse a actuar. En los testimonios tomados priman los principios de: incertidumbre, autoorganización y autonomía-dependencia, con lo cual se confirma que la vida no es estática y en ella las circunstancias no son determinísticas, la lectura que se hace de las situaciones y la cohesión de los colectivos para que se den estas dinámicas de trabajo.

\section{Reconocerse en el otro}

Reflexionar es preámbulo, vía y finalidad para comprender y apropiar los conocimientos, armonizar cualidades e intereses de las personas con las actividades que se les propone. Al mismo tiempo, es ponerse de cara a un proceso que exige desaprender lo que se había mecanizado y correr riesgos al preguntar, al ensayar posibles rutas de actuación, al interrelacionarse con personas formadas en disciplinas afines y diferentes. Visto ese panorama desde otro lugar, reconocerse en el otro es una apuesta por el desarrollo de las personas en los contextos de actuación. Es un desarrollo que escapa a la circunscripción esquemática de lo que es posible realizar, para dar cabida a las acciones que se realizan y que permiten aflorar capacidades diversas.

A veces a mí me cuesta entender lo que se está proponiendo, primero, porque venía de un estilo de trabajo en el que nos invitaban 
a hacer sin estudiar, sino partiendo de la experiencia, y segundo, porque por mi formación, a veces no entiendo los lenguajes utilizados (Informante 20).

No entiendo algunas decisiones porque a veces uno recibe una directriz, pero después viene una distinta, uno diría que acoge una de las dos, pero resulta que de todas se espera algún resultado (Informante 16).

Reconocer a los otros en sus distintos aspectos exige flexibilizar las relaciones, los lenguajes y la comunicación para hacer asequibles la información y los procesos; requiere también flexibilizarse a sí mismo, abrirse a los conocimientos e invita a considerar estilos particulares en las actuaciones y articular saberes para buscar transformaciones en procesos personales y organizacionales. A su vez, es una demanda que sugiere cuestionamiento a los esquemas disciplinares, no sólo de la formación académica, sino de las prácticas laborales, en las que ceñirse a lo propio de una manera de proceder, de un saber o de un estatuto epistemológico, puede limitar o impedir ver alternativas en el trabajo.

Complemento de lo anterior, irse por un saber de manera específica, por una parte, puede trozar errores presentados en los manejos disciplinares, administrativos o académicos de alguien; por otra parte, puede amputar borrosidades que enriquecerían de manera insigne las prácticas directivas docentes. Por lo dicho, reconocerse a partir de la mirada, el reconocimiento y la valoración del otro es reafirmarse como inter-dependiente, influido e influyente en las interacciones y en la consolidación de roles dentro de las organizaciones y de la sociedad.

Esta interrelación, denominada autonomía-dependencia, es refrendada con el siguiente testimonio:

Tenemos gente, tenemos procesos que hay que controlar y nos controlan, porque hay, digamos, parámetros de los cuales hay que cumplir y respetar, entonces, ante esos temas de dependencia, también de alguna manera respetan (Informante 9). 


\section{OTRAS REFLEXIONES}

Llamamos otras reflexiones a las pinceladas con que damos por concluido este escrito, no el tema. Al tratarse de un avance teórico de un tema poco explorado, el cual deja abierto un abanico de posibilidades para ahondar y hallar nuevas sinergias y borrosidades teóricoprácticas, sólo puede advertirse la constancia de las emergencias y de las dinámicas que exigirán otras decisiones y configuraciones a la gestión de la educación superior y en las instituciones de la sociedad en general. Por ello, son necesarias las siguientes dos acciones:

- Reconocer que, en la actualidad, prima el paradigma de la producción, pero esto no cancela la urgente necesidad de pasar, pedagógica y progresivamente en todas las áreas de gestión, al paradigma del aprendizaje, en el que se vinculan los cambios en las relaciones de estudiantes, docentes y comunidad en general, entre sí, con los otros, con el entorno y con los conocimientos. En ese orden de ideas, es también pasar hacia una gestión integradora e integral, con participación de diversidades humanas vinculadas para establecer mecanismos internos de evaluación, tendientes a cualificar-se ${ }^{2}$ como función en la totalidad y no en la suma de las partes.

- Entender que la gestión directiva universitaria, hoy más que nunca, está compelida a construir y vivenciar colegiadamente modelos educativos que promuevan un quehacer centrado en las capacidades de las personas, pero también orientado a aprovechar las oportunidades y necesidades de las comunidades donde se tiene presencia, creando un ecosistema universitario a partir del cual es posible construir propuestas y soluciones basadas en condiciones reales, en la cuales tenga cabida la incertidumbre, la auto-organización, la recursividad y la autonomía para superar un pensar-hacer exclusivamente prescriptivo, dogmático, lineal, axiomático y binario y consolidar prácticas innovadoras y pertinentes que faciliten caminar en el horizonte hacia una educación superior de alta calidad.

\footnotetext{
${ }^{2}$ La partición de la palabra es para indicar una acción de cada persona, al igual que los procesos, las funciones y los roles que asume.
} 
Concordante con el objetivo del artículo, en las prácticas de gestión de los Directivos docentes son identificables las siguientes tensiones generalizadas: a) la consideración de las particularidades a sus colaboradores vs la exigencia de rendir indicadores, y aunque no se trata de una oposición eficacia-eficiencia, queda una pregunta por el carácter de derecho o de servicio de la educación y por la productividad esperada de la misma; b) entre las dinámicas de los colaboradores de los directivos y las exigencias paramétricas externas, no sólo se presentan contradicciones, sino también desafíos transformados en oportunidades de cualificación individual y organizacional; c) si se pretendiera hacer teoría de las emergencias de un equipo de trabajo cualquiera, se aniquilarían cualidades como la iniciativa, la recursividad y la voluntariedad para el trabajo colaborativo.

El carácter cambiante de las personas y de los contextos no son cuestión de un paradigma determinado, sino expresión de una actitud y disposición propias de un pensamiento abierto, amplio y flexible, atento a las circunstancias cambiantes, a la modificabilidad y a la continua adaptación. La identificación de principios del paradigma emergente es una manera de referir atributos de las reflexiones realizadas en torno a una gestión tendiente a la corporatividad, más que a la individualidad.

Si las IES vuelven su mirada sobre aquellas prácticas que desarrollan sus directivos, consciente o inconscientemente, a través de las cuales se constituyen resultados de éxito, superando, en muchos casos, lo planeado o lo previsto desde la teoría, estarán abriendo un abanico de posibilidades para caminar, no sólo de forma más creativa, sino más acertadamente hacia la materialización de los retos, objetivos, indicadores de sus proyectos educativos, porque, como se pudo develar con este proyecto, la esencia de los buenos resultados no está dada por los objetos inanimados, sino por las relaciones utópicas que pueden ser creadas entre las personas, su entorno y el conocimiento. 


\section{REFERENCIAS BIBLIOGRÁFICAS}

Acosta, A. (1998). Cambio institucional y complejidad emergente de la educación superior en América latina. Revista Perfiles Latinoamericanos, 7(12), 109-140. Recuperado de http://perfilesla.flacso.edu.mx/ index.php/perfilesla/article/view/382/336.

Aguerrondo, I. (2014). Planificación educativa y complejidad: gestión de las reformas educativas. Cuadernos de Pesquisa, 44(153), 548-578.

Aguilera, M. A. (2011). Importancia y características de la función directiva. En La función directiva en secundarias públicas. Matices de una tarea compleja (pp. 11-229). México: INEE.

Assman, H. (2002). Placer y ternura en la educación. Hacia una sociedad aprendiente. Madrid: Narcea.

Aveiga, V., y Marín, C. (2013). La superación de la gestión académica del directivo universitario. Revista ECA Sinergia, 4(4), 89-100.

Ball, S. (2013). Performatividad y fabricaciones en la economía educacional: rumbo a una sociedad performativa. Pedagogía y Saberes, (38), 103-113.

Ballesteros, M. (2011). Pensamiento complejo y estudio de la comunicación. PHONICA, (7), 3-18. Recuperado de http://diposit.ub.edu/ dspace/bitstream/2445/110491/1/606205.pdf.

Bartra, R. (2011). Antropología del cerebro: Determinismo y libre albedrío. Salud Mental, 34(1), 1-9.

Bauman, Z. (2000). Modernidad líquida. México: Fondo de Cultura Económica.

Bateson, G. (1991). Pasos hacia una ecología de la mente. Buenos Aires: Lhole Lumen.

Boff, L., y Muraro, R. M. (2004). Femenino y masculino, una nueva consciencia para el encuentro de las diferencias. Madrid: Trotta.

Bohm, D.J. (1997). Sobre el diálogo. Barcelona: Kairós.

Boisier, S. (2001). Sociedad del conocimiento, conocimiento social y gestión territorial. Revista Internacional de Desenvolvimiento Local. Interaçôes, 3(2), 9-28.

Bolívar, A. (2010). El liderazgo educativo y su papel en la mejora: Una revisión actual de sus posibilidades y limitaciones. Psicoperspectivas, 9(2), 9-33. Recuperado de https://scielo.conicyt.cl/pdf/psicop/v9n2/ art02.pdf 
Cabanelas, D. T. (1993). Diccionario jurídico elemental. Buenos Aires: Heliasta. Recuperado de https:/es.calameo.com/ $\mathrm{read} / 001710011 \mathrm{c} 51 \mathrm{~d} 9009 \mathrm{ad} 9 \mathrm{e}$

Calvo, C. (2009). Los procesos educativos y la emergencia de complejidades caóticas y autoorganizadas. Revista Acción Pedagógica, (18), 6-19.

Capra, F. (1998). El punto crucial: Ciencia, sociedad y cultura naciente. Buenos Aires: Editorial Troquel.

Casassus, J. (2008). Problemas de la gestión educativa en América Latina: la tensión entre los paradigmas de tipo A y el tipo B. Aberto, 75(19), 49-69.

Castellano, L. A. y Mársico, C. T. (1995). Diccionario etimológico de términos usuales en la práctica docente. Buenos Aires: Altamira.

Cerri, Ch. (2015). Dependencia y autonomía: una aproximación antropológica desde el cuidado de los mayores. Athenea Digital, 15(2), 111140. Recuperando de http://dx.doi.org/10.5565/rev/athenea.1502

Chacón, M. L. (2014). Gestión educativa del siglo XXI: bajo el paradigma emergente de la complejidad. Omnia, 20(2), 150-161.

Chaverra, L. M., y Alzate, F. A. (2014). Comunidades académicas bioaprendientes: Emergencias autoorganizativas en el contexto de la educación superior. Revista Gestión de la educación, 2(4), 119-129.

Columbie, P. N. (2012). Un paradigma para la formación de una cultura ambiental. Letonia: Editorial Académica Española.

Corporación Universitaria Minuto de Dios. (2015). Compendio de Investigaciones. Bogotá: Centro editorial UNIMINUTO.

Díaz, P. J. A. (2013). Calidad educativa: un análisis sobre la acomodación de los sistemas de gestión de la calidad empresarial a la valoración en educación. Tendencias Pedagógicas, 21, 117-194.

Domínguez, G. (2001). La Sociedad del conocimiento y las organizaciones educativas como generadoras de conocimiento: el nuevo continuum cultural y sus repercusiones en las dimensiones de una organización. Revista Complutense de Educación, 12(2), 485-528.

Eisler, R. (1998). Placer Sagrado. Vol. II. Barcelona: Editorial Cuatro Vientos. Elizalde, A. (2003). Desarrollo Humano y Ética para la sustentabilidad. Panamá: Programa de las Naciones Unidas para el Medio Ambiente (PNU$M A)$, Oficina Regional para América Latina y el Caribe.

Elizondo, E. (2011). Competencias que debe tener un director académico universitario para la educación superior basada en competencias. Revista de investigación educativa, 29(1), 205-218. 
Fayol, H. (1931). Administration industrielle et générale: prevoyance. organisation, commandement, coordination; contrôle. París: H. Dunod y E. Pinat.

Flores, F. (1997). Creando organizaciones para el futuro. Santiago, Chile: Dolmen Ediciones.

Guarnizo, E. (2003). The Economics of Transnational Living. International Migration Review, 37(3), 666-699.

Gutiérrez, F. (2006). Doctorado de la Tercera Cultura. En busca del sentido. San José, Costa Rica: Nuestra Tierra.

Gutiérrez, F. (2008). Educación como praxis política. México: Siglo XXI.

Helmsing, B. (2001). Externalities, Learning and Governance: New Perspectives on Local Economic Development. Development and Change, 32, 277-308. doi:10.1111/1467-7660.00206

Jara, M. I. (2004). Emergencia y sorpresa. Causalidad o emergencia: diálogo entre filósofos y científicos. Bogotá: Universidad de la Sabana.

Lederman, L., y Teresi, D. (1996). La Partícula Divina. Barcelona: Crítica, Grijalbo Mondadori.

Luhmann, N. (1998). Sistemas Sociales. Lineamientos para una teoría general. Bogotá: CEJA, Pontificia Universidad Javeriana.

Lipton, B. (2007). La biología de la ciencia: La liberación del poder de la conciencia, la materia y los milagros. Madrid: Palmyra.

Maturana, H. (1992). El sentido de lo humano. Santiago, Chile: Ediciones Pedagógicas Chilenas.

Mayo, E. (1977). The human problems of an industrial civilization. Nueva York: Arno.

Morales, M. E., Sanabria, P. E., y Caballero, D. (2014). Características de la vinculación universidad-entorno en la Universidad Nacional de Colombia. Rev.fac.cienc.econ., XXIII(1), 189-208.

Morin, E. (1999). Los siete saberes necesarios para la educación del futuro. París: UNESCO.

Morin, E., Roger-Ciurana, E., y Motta, R. (2003). Educar en la era planetaria. Salamanca: Gráficas Varona.

Mussa, J. A., Morales V. A., y Martínez, E. M. (2011). Cognición Cuántica. El aprendizaje emocional y la creatividad desde la física cuántica. En Congreso Internacional de Teoría de la Educación. Congreso llevado a cabo en la Universidad de Barcelona, España.

Najmanovich, D. (2008). Mirar con ojos nuevos. Nuevos paradigmas en la ciencia y pensamiento complejo. Buenos Aires: Biblos. 
Navarro, A. (1997). La gerencia social y el nuevo paradigma de gerencia. Revista Costarricense de Trabajo Social, (7), 1-24. Recuperado de http://www.ts.ucr.ac.cr/binarios/docente/pd-000170.pdf.

Novo, M. (2007). Mujer y medio ambiente. Los caminos de la visibilidad: Utopias, educación y nuevo paradigma. Madrid: Catarata.

Payán, J. (2000). Lánzate al vacio, se extenderán tus alas. Bogotá: McGraw Hill.

Pigem, J. (1991). Nueva conciencia. Barcelona: Integral.

Ramírez, Á. V. (2012). Gobierno abierto es la respuesta: ¿Cuál era la pregunta? Más poder local, (Especial), 14-22.

Razeto-Barry, P., y Ramos-Jiliberto, R. (2013). ¿Qué es autopoiesis? En Autopoiesis. Un concepto vivo (pp. 27-57). Santiago, Chile: Editorial Nueva Civilización.

Tapiero-Vásquez, E., y García-Quiroga, B. (2008). La teoría neosistémica y el desarrollo institucional. Educación y Educadores, 2(11), 213-226.

Taylor, F. (1911). Scientific management. Nueva York: Harper and Row.

Tünnermann, C. (2006). Pertinencia y calidad de la educación superior. Guatemala: Universidad Rafael Landívar. Recuperado de http://iep.udea. edu.co:8180/jspui/bitstream/123456789/258/1/Pertinencia\%20Calidad\%20Educacion\%20Superior\%20-CarlosTunnermann.pdf

Ugarte, D. (2007). El poder de las Redes. Manual ilustrado para personas, colectivos y empresas abocados al ciberactivismo. Madrid: Ediciones El Cobre.

Universidad Católica de Oriente. (2016). Plan de Desarrollo Institucional 2016-2025. Rionegro: Fondo Editorial Universidad Católica de Oriente.

Verlee, L. (2008). Aprender con todo el cerebro. Estrategias y modos de pensamiento visual, metafórico y multisensorial. Revista de Estudios Interdisciplinarios en Ciencias Sociales, 3(10), 465-467. 